\title{
A Comparison of the Effects and Side Effects of Oral Betahistine with Injectable Promethazine in the Treatment of Acute Peripheral Vertigo in Emergency
}

\author{
Hassan Motamed ${ }^{\mathrm{a}}$, Meisam Moezzi ${ }^{\mathrm{a}, \mathrm{c}}$, Ali Dalir Rooyfard ${ }^{\mathrm{a}}$, \\ Kambiz Ahmadi Angali ${ }^{b}$, Zahra Izadi ${ }^{\mathrm{a}}$
}

\begin{abstract}
Background: Vertigo is an illusion of rotation that is caused by the asymmetry of neurological function of the right and left vestibular nuclei. The aim of this study was to compare the efficacy and side effects of oral betahistine with injectable promethazine in treatment of acute peripheral vertigo.
\end{abstract}

Methods: In this double-blind clinical trial study, the patients with acute peripheral vertigo were assigned in the two groups: receiving promethazine intramuscularly at a dose of $25 \mathrm{mg}$ (group A) and receiving $8 \mathrm{mg}$ betahistine tablets (group B) and the severity of their vertigo was evaluated on the visual analog scale (VAS) scoring system. In addition, adverse events in both groups of patients were compared and evaluated.

Results: A total of 162 patients (82 subjects in group A) participated in this study. The mean age, gender distribution, intensity and symptoms of vertigo were similar in both groups before the intervention. At 2 and $3 \mathrm{~h}$ after the intervention, the score of VAS in patients taking betahistine was significantly higher than promethazine. In addition, the clinical symptoms after taking betahistine were significantly less. Side effects seen in patients taking the promethazine include mainly drowsiness. After taking betahistine, the most common complication was abdominal problems (nausea and vomiting).

Conclusion: The results of this study show that betahistine is a safe and effective drug in controlling patients with acute vertigo and its impact is more than promethazine.

Keywords: Betahistine; Promethazine; Acute peripheral vertigo

Manuscript submitted June 6, 2017, accepted October 11, 2017

aDepartment of Emergency Medicine, Ahvaz Jundishapur University of Medical Sciences, Ahvaz, Iran

bepartment of Biostatistics and Epidemiology, Faculty of Health, Ahvaz Jundishapur University of Medical Sciences, Ahvaz, Iran

${ }^{\mathrm{c} C}$ Corresponding Author: Meisam Moezzi, Department of Emergency Medicine, Ahvaz Jundishapur University of Medical Sciences, Ahvaz, Iran.

Email: meisam.moezzi@yahoo.com

doi: https://doi.org/10.14740/jocmr3093w

\section{Introduction}

Vertigo is an illusion of rotation that is caused by the asymmetry of neurological function of the right and left vestibular nuclei [1]. The differential diagnosis of vertigo is vital for various reasons; first of all, the vertigo can be appeared as a lifethreatening situation to a normal physiological response and secondly, there is no definitive diagnostic tool for the detection of its reasons [2]. Therefore, proper management of these patients in health centers is always challenging. However, the vertigo is a common cause for referring patients to emergency centers. So that it is estimated that its prevalence among young people and in adults is $1.8 \%$ and $30 \%$, respectively, and it is a reason for referring $4 \%$ of all patients presenting to emergency centers $[3,4]$. Patients due to vertigo usually fall into one of the three groups: 1) acute severe vertigo, 2) recurrent attacks of vertigo, and 3) recurrent positional vertigo. The most common cause in all three groups is benign peripheral vestibular dysfunction [5].

Pharmacological treatments of vertigo are divided into two groups: symptomatic and causal treatments. In the sysmptomatic treatments, neuroleptics drugs, anxiolytics and firstgeneration antihistamines are used that affect the medulla, hypothalamus and limbic system and cause reduction in the neurovegetative symptoms (nausea, vomiting, palpitations, sweating and anxiety) [6]. Antihistamines include the H1-H4 histamine receptor antagonist medications and are widely used in the prevention and treatment of vertigo symptoms. H1 histamine receptor antagonists currently are most used in patients with vertigo, and there are several drugs, including meclizine, astemizole and promethazine in this pharmacological group [7]. The usefulness of these drugs in treating vertigo is due to block histaminergic signals from the vestibular nucleus to the vomiting center in the medulla [8]. This is despite the fact that recently some evidence has shown that in addition to histamine $\mathrm{H} 1$ receptor antagonists, betahistine may also improve effective vertigo symptoms $[9,10]$. Betahistine is a structural analogue of histamine with pharmacological characteristics similar to it. Betahistine is as an $\mathrm{H} 1$ receptor agonist and a relatively strong antagonist of the $\mathrm{H} 3$ receptor [11]. The effect of betahistine on vertigo is due to the improved vestibular performance by increasing blood circulation in the inner ear and the vasodilator function [7]. 
Some side effects of antagonists $\mathrm{H} 1$, such as their sedative effect, have led to limitation of their use in the treatment of patients with vertigo. However, the betahistine does not have this sedative effect [11]. So if the effect of these two drugs is same, betahistine can be an appropriate alternative in the treatment of patients with vertigo. However, so far no studies have been conducted for comparing the two drugs in improving vertigo symptoms. So, the study aimed to evaluate the effect of betahistine compared with promethazine in patients with acute peripheral vertigo.

\section{Materials and Methods}

\section{Study design}

In this double-blind clinical trial study, patients, who had been referred to Imam Khomeini Hospital, Ahvaz, Iran, due to peripheral vertigo, were studied. Inclusion criteria for the study were: age above 18 years and complaints of vertigo. While pregnant patients, history of allergy to studied drugs, antiemetic use in the last $24 \mathrm{~h}$ and patients with traumatic brain injury were excluded from the study. Demographic data, time of onset and duration of symptoms, associated symptoms (nausea, vomiting, tinnitus and headaches), changes in hearing, neurological history, medications, recent otitis history and head trauma were questioned and recorded in a questionnaire. This study was approved by the ethics committee of Ahvaz Jundishapur University of Medical Sciences and patients signed a written informed consent and then, were enrolled.

\section{Evaluation of patients}

All patients underwent physical examination (neurological and neurotological), including: complete bed-side vestibular test, Dix-Hallpike maneuver, Rinne and Weber test, oculomotor examination, positional test, head thrust test, Romberg's test, tandem gait test, and Fukuda stepping test.

\section{Therapeutic intervention}

Patients eligible were randomly selected by removing the sealed envelopes containing the treatment type available in emergency pharmacies. The drugs used were placed in separate boxes containing $25 \mathrm{mg}$ of promethazine injection, placebo tablets and betahistine tablets $8 \mathrm{mg}$. Patient and physician have not been informed of what treatment each patient has received. Moreover, the person that measured the answers did not know which group each patient is in. So, blinded design was done successfully. Randomization code was preserved until time of record analysis of data. One package, including a form for receiving records and promethazine or betahistine drugs, was used for each patient.

Group A received a dose of $25 \mathrm{mg}$ promethazine intramuscularly and group B received tablets of $8 \mathrm{mg}$ betahistine orally. Medication dose was used similar to the standard dose
Table 1. Patient's Characteristics

\begin{tabular}{clll}
\hline Variables & Group A $(\mathbf{n}=\mathbf{8 2})$ & Group B $(\mathbf{n}=\mathbf{8 0})$ & P value \\
\hline $\begin{array}{c}\text { Age (range) } \\
\text { Gender }\end{array}$ & $43.5(19-64)$ & $40.1(17-66)$ & NS \\
Female & $45(54.9 \%)$ & $42(52.5 \%)$ & NS \\
Male & $37(45.1 \%)$ & $38(47.5 \%)$ & NS \\
\hline
\end{tabular}

routinely used in our department.

\section{Measurements}

Before treatment, the baseline of vertigo of participants was ranked by horizontal visual analog scale (VAS). VAS ranking has two ranges: 0 equals to no vertigo, and 10 means the worst possible vertigo [12]. Effectiveness of each drug was assessed by VAS changes. VAS test was done consecutively for each hour up to $3 \mathrm{~h}$ after drug administration.

All adverse events during the study period were recorded, according to a report by the treating physician or the patient in a recorded form. Then the complications were compared in each group.

\section{Data analysis}

First, the data obtained were analyzed in terms of descriptive indexes and then to compare quantities between the two groups, according to data normality, $t$-test and Mann-Whitney test were used. Qualitative data were analyzed by the Chisquare test. All statistical analyses were performed using the SPSS. A significant $P$ value was considered as less than 0.05 .

\section{Results}

From April 2015 to April 2016, 162 patients (group A = 82 and group $\mathrm{B}=80$ ) were studied, of which $53.7 \%$ were male. Patients were in the age range from 18 to 65 years; their mean age was $41.8 \pm 13.6$. Their gender distribution and average age of the patients were similar in both groups (Table 1).

The VAS level before the intervention and $1 \mathrm{~h}$ after treatment showed no significant difference between both groups. However, the rate at 2 and $3 \mathrm{~h}$ after the intervention in group A (taking promethazine) was higher (Table 2). Clinical symptoms of patients before the intervention were relatively similar. However, at times of 1,2 and $3 \mathrm{~h}$ after the intervention, the prevalence of clinical symptoms in both groups showed a different distribution that was statistically significant. Generally, in all three time points, the number of patients with no clinical symptoms in group B (taking betahistine) was higher. Also in group B, nausea and vomiting were observed more frequently, but in group A, drowsiness was observed as the most common presentation (Table 3). Unwanted drug side effects were observed only in group A, the most common drowsiness (76.8\%) followed by akathisia. But in the group B these adverse effects 
Table 2. Comparison of Pain Level at Different Times in Both Group A and B

\begin{tabular}{lllll}
\hline Variable & Time & Group A $(\mathbf{n}=\mathbf{8 2})$ & Group B $(\mathbf{n}=\mathbf{8 0})$ & P value \\
\hline VAS $($ mean $\pm \mathrm{SD})$ & Before intervention & $6.26 \pm 1.22$ & $6.44 \pm 0.6$ & NS \\
& $1 \mathrm{~h}$ & $5.09 \pm 1.39$ & $4.84 \pm 1.14$ & $\mathrm{NS}$ \\
& $2 \mathrm{~h}$ & $4.28 \pm 1.3$ & $3.38 \pm 0.98$ & 0.043 \\
Vertigo after intervention $(\mathrm{h})$ & $3.39 \pm 1.3$ & $2.7 \pm 0.75$ & 0.039 \\
\hline
\end{tabular}

VAS: visual analog scale.

were not observed (Table 4).

\section{Discussion}

Vertigo is a common cause for referring patients to emergency centers and its consequences are in a wide range; on the one hand, they affect life-threatening conditions and on the other hand, they affect normal physiological response [2-4]. Treatment of patients has been often symptomatic and different drug groups are used to control it. Histamine antagonists are one of the most commonly used drugs in improving vertigo symptoms, which sometimes makes a lot of unwanted side effects such as sedative effects in patients and limits their use. Hence the attempt to find appropriate alternative with similar efficacy and fewer side effects continues. In accordance with the evidence that shows betahistine has the mentioned features (high efficacy and fewer side effects), in this study, betahistine was compared with promethazine (H1 receptor antagonist) in patients with vertigo. The results of this study show that while VAS levels decreased in both groups, the amount measured at the time points of 2 and $3 \mathrm{~h}$ after the intervention therapy in patients receiving betahistine was significantly less. So, according to these findings, it seems that betahistine has been more effective than promethazine in improving symptoms of vertigo. However, according to our knowledge, there is no similar study that has assessed the effects of betahistine and promethazine on vertigo, but comparison between betahistine with placebo and other drugs used for vertigo results confirm the results of this study. In a double-blind trial study, Oosterveld et al assessed the effect of betahistine on improving the vertigo in 24 patients, and showed that the severity of vertigo and also nausea and vomiting after taking betahistine has been significantly decreased [13]. Similarly, in a study conducted on 144 patients with vertigo, Simoncelli et al assessed the symptoms influence in the betahistine examined and showed that the frequency, severity and duration of attacks of vertigo in patients taking betahistine were significantly less, and also the quality of life before and after using the drug significantly improved [14]. In a randomized clinical trial, Alberta et al compared the effectiveness of betahistine with flunarizine in 52 patients with recurrent vertigo. In this study, the criterion dizziness handicap inventory (DHI) was used to compare the effects of both drugs. The results of this study showed that in

Table 3. Clinical Pictures During the Study in Group A and Group B

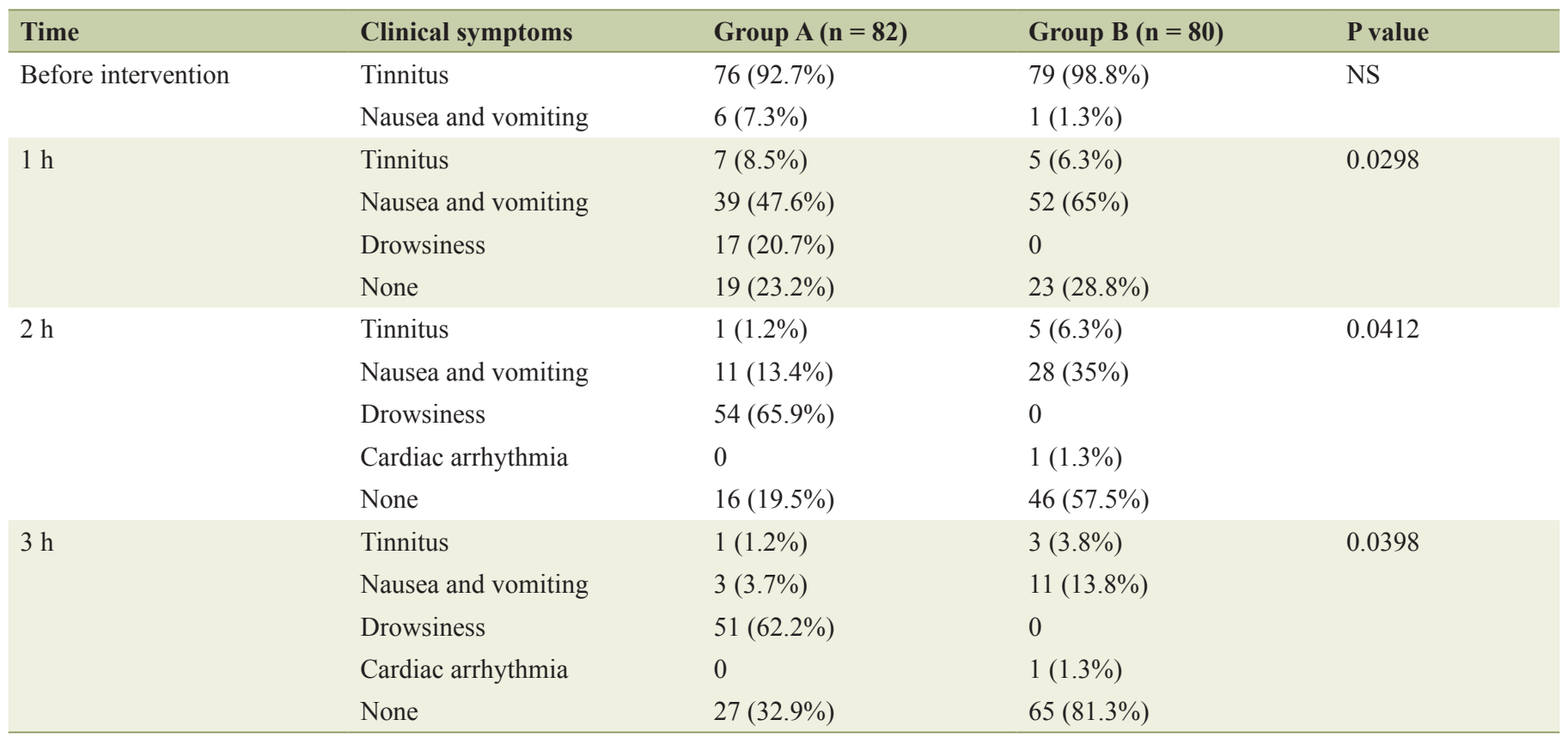


Table 4. Adverse Effect Incidence in Both Group A and Group B

\begin{tabular}{llll}
\hline Adverse effect & Group A $(\mathbf{n}=\mathbf{8 2})$ & Group B $(\mathbf{n = 8 0})$ & P value \\
\hline Restlessness & $11(13.4 \%)$ & 0 & 0.038 \\
Anxiety & $2(2.4 \%)$ & 0 & \\
Drowsiness & $63(76.8 \%)$ & 0 & \\
None & $6(7.3 \%)$ & $80(100 \%)$ & \\
\hline
\end{tabular}

patients taking betahistine, DHI was significantly lower than flunarizine and therefore betahistine has been more effective in improving symptoms of vertigo [15]. In a similar study, Fraysse et al confirm the recent findings [16]. This is despite the fact that on the contrary, it, Elbaz et al, have shown that flunarizine in improving vertigo is more effective than betahistine [17]. In addition, in the study of 88 patients with peripheral vertigo of unknown causes, Dearing et al compared the effect of betahistine with cinnarizine and showed that although both drugs have the same effect in reducing the severity and duration of attacks of vertigo, patients taking betahistine with less frequency are affected by vertigo attacks [18].

In connection with the safety of betahistidine, symptoms of the patients after taking the medication and its unwanted side effects compared with promethazine were studied. The results showed that while no effects of drowsiness, akathisia and anxiety were seen in patients taking betahistine, but the numbers of patients with the symptoms of nausea and vomiting in this group were higher than those receiving promethazine. These results are consistent with other studies $[13,16,18]$.

\section{Conclusions}

Overall, the findings of this study show that betahistine is more effective than promethazine in improving vertigo, and it has less unwanted side effects. Comparison of the effects of betahistine and promethazine, in patients with vertigo for the first time as well as the high sample size were some of the strengths of this study. While evaluating different doses of the drug and to determine the optimal dose as well as long-term follow-up of patients were some of the limitations of this study.

\section{References}

1. Halmagyi GM. Diagnosis and management of vertigo. Clin Med (Lond). 2005;5(2):159-165.

2. Jung I, Kim JS. Approach to vertigo in the emergency department. Clinical and Experimental Emergency Medicine. 2015;2(2):75-88.

3. Shahrami A, Norouzi M, Kariman H, Hatamabadi HR, Arhami Dolatabadi A. True vertigo patients in emergency department; an epidemiologic study. Emerg (Tehran). 2016;4(1):25-28.

4. Lam JM, Siu WS, Lam TS, Cheung NK, Graham CA,
Rainer TH. The epidemiology of patients with vertigo in an emergency department. Hong Kong J Emerg Med. 2006;13(3):133-139.

5. Kerber KA. Vertigo and dizziness in the emergency department. Emerg Med Clin North Am. 2009;27(1):39-50, viii.

6. Zatonski T, Temporale H, Holanowska J, Krecicki T. Current views on treatment of vertigo and dizziness. J Med Diagn Meth. 2014;3:150.

7. Amini A, Heidari K, Kariman H, Taghizadeh M, Hatamabadi H, Shahrami A, Derakhshanfar H, et al. Histamine antagonists for treatment of peripheral vertigo: a metaanalysis. J Int Adv Otol. 2015;11(2):138-142.

8. Simons FE, Simons KJ. H1 antihistamines: current status and future directions. World Allergy Organ J. 2008;1(9):145-155.

9. Lacour M. Betahistine treatment in managing vertigo and improving vestibular compensation: clarification. J Vestib Res. 2013;23(3):139-151.

10. Ramos Alcocer R, Ledezma Rodriguez JG, Navas Romero A, Cardenas Nunez JL, Rodriguez Montoya V, Deschamps JJ, Liviac Ticse JA. Use of betahistine in the treatment of peripheral vertigo. Acta Otolaryngol. 2015;135(12):1205-1211.

11. Lacour M, Sterkers O. Histamine and betahistine in the treatment of vertigo: elucidation of mechanisms of action. CNS Drugs. 2001;15(11):853-870.

12. Dannenbaum E, Chilingaryan G, Fung J. Visual vertigo analogue scale: an assessment questionnaire for visual vertigo. J Vestib Res. 2011;21(3):153-159.

13. Oosterveld WJ. Betahistine dihydrochloride in the treatment of vertigo of peripheral vestibular origin. A double-blind placebo-controlled study. J Laryngol Otol. 1984;98(1):37-41.

14. Mira E, Guidetti G, Ghilardi L, Fattori B, Malannino N, Maiolino L, Mora R, et al. Betahistine dihydrochloride in the treatment of peripheral vestibular vertigo. Eur Arch Otorhinolaryngol. 2003;260(2):73-77.

15. Albera R, Ciuffolotti R, Di Cicco M, De Benedittis G, Grazioli I, Melzi G, Mira E, et al. Double-blind, randomized, multicenter study comparing the effect of betahistine and flunarizine on the dizziness handicap in patients with recurrent vestibular vertigo. Acta Otolaryngol. 2003;123(5):588-593.

16. Fraysse B, Bebear JP, Dubreuil C, Berges C, Dauman R. Betahistine dihydrochloride versus flunarizine. A doubleblind study on recurrent vertigo with or without cochlear syndrome typical of Meniere's disease. Acta Otolaryngol Suppl. 1990;490:1-10.

17. Elbaz P. Flunarizine and betahistine. Two different therapeutic approaches in vertigo compared in a double-blind study. Acta Otolaryngol Suppl. 1988;460:143-148.

18. Deering RB, Prescott P, Simmons RL, Downey LJ. A double-blind crossover study comparing betahistine and cinnarizine in the treatment of recurrent vertigo in patients in general practice. Curr Med Res Opin. 1986;10(4):209214. 